\title{
Efficacy and safety of Bosutinib in Patient with Chronic myeloid leukemia who was intolerant to DASTANIB,NILOTUNIB -Case
} report

\author{
Abdullah Mohammad Arshad ${ }^{1}$ and mohamed yassin ${ }^{2}$ \\ ${ }^{1}$ Hamad Medical Corporation \\ ${ }^{2} \mathrm{HMC}$ NCCCR
}

September 5, 2021

\begin{abstract}
Bosutinib is approved as first line therapy for treatment of chronic phase CML and also in patients who are either resistant or intolerant to previous TKI. We present a 59 year old male who was intolerant to 2 TKI but showed excellent hematological and major molecular response to Bosutinib.
\end{abstract}

\section{Introduction}

Chronic myeloid leukemia is a myeloproliferative disorder which is characterized by increase amplification of the granulocytic cell line with fairly normal differentiation. The main hallmark of CML is deregulated tyrosine kinase activity catalyzed by BCR/ABL1 fusion protein which results on Philadelphia chromosome(1).

Treatment of CML has been revolutionized with the presence of BCR-ABL1 targeting TKI, which has improved the overall survival and quality of patient's life. Imatinib was the first of these to be used in the treatment of $\mathrm{CML}(2)$..Several other tyrosine kinase inhibitors (TKIs) have been developed and tested in patients with CML, most notably dasatinib, nilotinib, bosutinib, and ponatinib. Despite the excellent outcome, half of the patients will eventually fail due to intolerance or resistance to first line treatment, with many of them requiring a third or even further lines of therapy $(3,4)$.

We report a case of CML who initially didn't tolerate dasatinib and Nilotinib but showed excellent response to Bosutinib and achieved major molecular response in just 4 months of therapy.

\section{Case presentation}

59 year old gentleman with background of Diabetes and hypertension was diagnosed as a case of chronic myeloid leukemia in the month of April 2018. He was initially started on hydroxyurea for cytoreduction that he received for 2 weeks, afterwards switched to second generation tyrosine kinase inhibitor Dasatinib 100mg/d for 4 weeks. It was terminated because of severe diarrhea. He was then started on Nilotinib, that was also poorly tolerated because of worsening of his diabetes and persistent nausea. Bosutinib, was introduced in june 2018 and was continued till date. Not only he tolerated it well but also showed an exceptionally good, above average response MMR within 4 months. He didn't suffer from common side effects related to bosutinib such as Diarrhea, pleural effusion or Qtc prolongation.

\section{Discussion}

When it comes to managing patient with CML, the main objectives are to achieve clinical remission, halt disease progression and also providing optimal care for treatment related side effects. In practice so far there 
are five TKI approved which can be use in chronic phase namely Imatinib, Dasatinib, Nilotininb, Bousitinb, Ponatinib(5).Choice of initial TKI depends upon drug's toxicity profile, patient co morbid condition as tolerability of side effects depends upon it.

Imatinib was the very first drug approved for newly confirmed case of CML, But, an intention-to-treat analysis demonstrated that approximately $1 / 3 \mathrm{rd}$ of patients treated with diagnosed CML-CP showed inadequate responses or do not experience long-term benefit(6).

Dasatinib is a second line TKI, and considered to be more potent in achieving early molecular response as compare to first line Imatinib(7).It has been approved as first line therapy after DASISION trial(8).Common side effects are cytopenias, GI toxicity including diarrhea ,pleural effusion and Qtc prolongation .Our patient was initially started on Dasatinib as upfront therapy with 100mg daily for four weeks. But it was discontinued because of poor intolerance as he developed severe Diarrhea not responding to symptomatic management. As the patient is already diabetic and immunocompromised because of risk of sever colitis it was decided to terminate Dasatinib. Cases of Dasatinib induced CMV hemorrhagic colitis have been reported in past(9) .

Nilotinib, another $2^{\text {nd }}$ generation TKI is more potent than imatinib, inhibits several imatinib-resistant BCR-ABL1 mutants. It also comes with side effects such as cytopenias, hepatotoxicity, QTc prolongation, pancreatitis, hyperglycemia and long-term Cardiovascular complications(10).Not only it can worsen the preexisting Diabetes mellitus but association of directly causing diabetes have also been demonstrated in the literature $(11,12)$.In our patient he experienced worsening of his preexisting diabetes that lead to termination of nilotinib in his case.

Bosutinib is a second-generation TKI approved for the treatment of chronic-, accelerated-, and blast-phase CML in patients who are intolerant or resistant to prior therapy(13).

Results from BEFORE trial have successfully demonstrated patients who received bosutinib had significantly higher rates of major molecular response (MMR) and complete cytogenetic response (CCyR) by 12 and 24 months, and achieved responses faster, compared with imatinib-treated patients, for example, MMR and CCyR rates at 12 months were 47 versus $37 \%$ and 77 versus $66 \%$ for bosutinib and imatinib, respectively(14).

Bosutinib is an excellent choice for patients who developed resistance or showed intolerance to other TKI. Phase 4 BYOND study showed the efficacy of Bousitinb in achieving higher rates of not only cytogenetic but also molecular responses those with $\mathrm{Ph}+$ chronic phase $\mathrm{CML}$ and with excellent response in patient's resistant or tolerant to previous TKI(15).

It not only inhibit the activity of the BCR-ABL kinase but also exhibits activity against Src kinases, which are involved with malignant cell transformation, tumor progression, and metastasis(16).

Bosutinib is one the expensive drug, but affordability was not the issue in his case. He was started on Bosutinib 500mg daily. He achieved major molecular response MR3, bcr-abl/abl ratio 0,013\% a(s seen in figure 1)after only 4 months of initiating therapy.

Side effect profile of Bosutinib ranges from mild to moderate which can be managed by symptomatic management dose reduction or temporary stoppage of the drug(17). GI toxicity is the most common non hematological adverse effects in which diarrhea remains the top most which can occur early in the treatment .It is usually mild and can be manage with anti-diarrheal medications(17). Other includes, pleural effusion which can occur not only in patient's who had previous history of pleural effusion secondary to other TKI(Dasatinib) but also those without any past history. It is usually managed by either reducing the dose or sometime needs interruption of therapy(17).Cardiac and vascular events overall are less in long run in patients treated with bosutinib ,occur mainly in those with preexisting cardiac conditions $(17,18)$. Myelosuppression is the most common hematological side effect, in which thrombocytopenia is the most frequent.

Our patient tolerated Bosutinib very well as compare to previous TKI .He did experience diarrhea but the severity was less and controlled well with anti-diarrheal. 
In conclusion, each TKI come with its on toxicity profile as this needs to be taken in account before starting therapy with particular agent in a patient. Most of the adverse effects related to TKI are mild and can be managed by either symptomatic treatment or either by dose reduction. But some patients can become intolerant and to switch to other TKI remains the only option.Bosutinib can be an excellent choice in not only achieving good molecular and hematological response but also a good option in those who experience intolerance to previous TKI.

\section{Declarations}

\section{Ethical approval and consent}

Consent was obtained from the patient. The hospital's local IRB approval was sought for this case report publication.

\section{Data availability}

Further details about the case are available upon request.

\section{Conflict of interest}

The authors have no conflict of interest relevant to this case.

\section{Author contribution}

Abdullah Mohammad Arshad: has written the initial draft of the manuscript and attempted literature review Mohamed A Yassin: updated and did revision of draft

Both authors critically reviewed the initial and final draft of the manuscript and approved it for submission

\section{Acknowledgements}

I wish to show my gratitude to the Internal medicine resi-

dency program, to Dr Dabia Hamad Almohanadi, and I

would like to thank the patient for allowing us to share his

case

References

1. Turkina A, Wang J, Mathews V, Saydam G, Jung CW, Al Hashmi HH, et al. TARGET: a survey of real-world management of chronic myeloid leukaemia across 33 countries. Br J Haematol. 2020.

2. Al-Dewik NI, Morsi HM, Samara MM, Ghasoub RS, Gnanam CC, Bhaskaran SK, et al. Is Adherence to Imatinib Mesylate Treatment Among Patients with Chronic Myeloid Leukemia Associated with Better Clinical Outcomes in Qatar? Clin Med Insights Oncol. 2016;10:95-104.

3. Vezzalini M, Mafficini A, Tomasello L, Lorenzetto E, Moratti E, Fiorini Z, et al. A new monoclonal antibody detects downregulation of protein tyrosine phosphatase receptor type gamma in chronic myeloid leukemia patients. J Hematol Oncol. 2017;10(1):129.

4. Al-Dewik NI, Jewell AP, Yassin MA, El-Ayoubi HR, Morsi HM. Studying the impact of presence of point mutation, insertion mutation and additional chromosomal abnormalities in chronic myeloid leukemia patients treated with imatinib mesylate in the State of Qatar. QScience Connect. 2014;2014(1).

5. Mughal TI, Radich JP, Deininger MW, Apperley JF, Hughes TP, Harrison CJ, et al. Chronic myeloid leukemia: reminiscences and dreams. Haematologica. 2016;101(5):541-58.

6. de Lavallade H, Apperley JF, Khorashad JS, Milojkovic D, Reid AG, Bua M, et al. Imatinib for newly diagnosed patients with chronic myeloid leukemia: incidence of sustained responses in an intention-to-treat analysis. J Clin Oncol. 2008;26(20):3358-63. 
7. Cortes JE, Saglio G, Kantarjian HM, Baccarani M, Mayer J, Boque C, et al. Final 5-Year Study Results of DASISION: The Dasatinib Versus Imatinib Study in Treatment-Naive Chronic Myeloid Leukemia Patients Trial. J Clin Oncol. 2016;34(20):2333-40.

8. Kantarjian HM, Shah NP, Cortes JE, Baccarani M, Agarwal MB, Undurraga MS, et al. Dasatinib or imatinib in newly diagnosed chronic-phase chronic myeloid leukemia: 2-year follow-up from a randomized phase 3 trial (DASISION). Blood. 2012;119(5):1123-9.

9. Yassin MA, Nashwan AJ, Soliman AT, Yousif A, Moustafa A, AlBattah A, et al. Cytomegalovirusinduced Hemorrhagic Colitis in a Patient with Chronic Myeloid Leukemia (Chronic Phase) on Dasatinib as an Upfront Therapy. Clin Med Insights Case Rep. 2015;8:77-81.

10. Hochhaus A, Saglio G, Hughes TP, Larson RA, Kim DW, Issaragrisil S, et al. Long-term benefits and risks of frontline nilotinib vs imatinib for chronic myeloid leukemia in chronic phase: 5-year update of the randomized ENESTnd trial. Leukemia. 2016;30(5):1044-54.

11. Ito Y, Miyamoto T, Chong Y, Maki T, Akashi K, Kamimura T. Nilotinib exacerbates diabetes mellitus by decreasing secretion of endogenous insulin. Int J Hematol. 2013;97(1):135-8.

12. Ismail AS, Yassin MA. Nilotinib-Induced Diabetes Mellitus in a Young Female with Chronic Myeloid Leukemia. Cureus. 2020;12(9):e10277.

13. Doan V, Wang A, Prescott H. Bosutinib for the treatment of chronic myeloid leukemia. Am J Health Syst Pharm. 2015;72(6):439-47.

14. Cortes JE, Gambacorti-Passerini C, Deininger MW, Mauro MJ, Chuah C, Kim DW, et al. Bosutinib Versus Imatinib for Newly Diagnosed Chronic Myeloid Leukemia: Results From the Randomized BFORE Trial. J Clin Oncol. 2018;36(3):231-7.

15. Hochhaus A, Gambacorti-Passerini C, Abboud C, Gjertsen BT, Brummendorf TH, Smith BD, et al. Bosutinib for pretreated patients with chronic phase chronic myeloid leukemia: primary results of the phase 4 BYOND study. Leukemia. 2020;34(8):2125-37.

16. Keller VAG, Brummendorf TH. Novel aspects of therapy with the dual Src and Abl kinase inhibitor bosutinib in chronic myeloid leukemia. Expert Rev Anticancer Ther. 2012;12(9):1121-7.

17. Kantarjian HM, Cortes JE, Kim DW, Khoury HJ, Brummendorf TH, Porkka K, et al. Bosutinib safety and management of toxicity in leukemia patients with resistance or intolerance to imatinib and other tyrosine kinase inhibitors. Blood. 2014;123(9):1309-18.

18. Cortes JE, Jean Khoury H, Kantarjian H, Brummendorf TH, Mauro MJ, Matczak E, et al. Long-term evaluation of cardiac and vascular toxicity in patients with Philadelphia chromosome-positive leukemias treated with bosutinib. Am J Hematol. 2016;91(6):606-16.

\section{Number of figures-1}


BCR-ABL1 Expression (Figure-1)

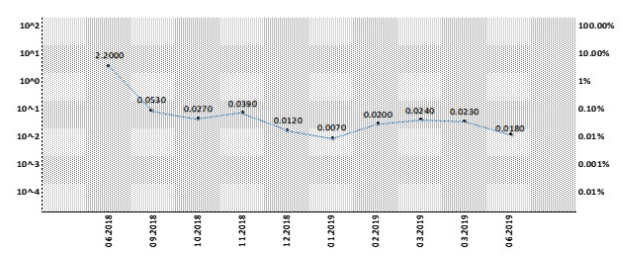

